\title{
Two Level Finite Element Technique for Pressure Recovery from Stream Function Formulation of The Navier-Stokes Equations
}

\author{
Faisal Fairag \\ Mathematics Department, King Fahd University of Petroleum \& Minerals, \\ Dhahran, 31261 Saudi Arabia (ffairag@kfupm.edu.sa).
}

\begin{abstract}
We consider two-level finite element discretization methods for the stream function formulation of the Navier-Stokes equations. The two-level method consists of solving a small nonlinear system on the coarse mesh, then solving a linear system on the fine mesh. It is shown in [8] that the errors between the coarse and fine meshes are related superlinearly. This paper presents an algorithm for pressure recovery and a general analysis of convergence for the algorithm. The numerical example for the 2D driven cavity fluid is considered. Streamfunction contours are displayed showing the main features of the flow.
\end{abstract}

\section{Introduction}

Two level finite element method is one of the promising approaches for solving nonlinear problems that arise in areas such as fluid mechanics. The computational attractions of the methods are that they require the solution of only a small system of nonlinear equations on coarse mesh and one linear system of equations on fine mesh. Apparently, the two-level method was proposed first in $[16,15,14]$ and used for semilinear elliptic problems. The method was implemented for the velocity-pressure formulation of the Navier-Stokes equations in [11-13] and for the streamfunction formulation of the Navier-Stokes equations in $[8,17]$.

The Navier-Stokes equations may be solved using either the primitive variable or stream function formulation. Here, we use the stream function formulation. The attractions of the stream function formulation are that the incompressibility constraint is automatically satisfied, the pressure is not present in the weak form, and there is only one scalar unknown to solve for. The standard weak formulation of the stream function version first appeared in 1979 in [10]. In this direction, Cayco and Nicolaides [6,5,4] studied a general analysis of convergence for this standard weak formulation. Once the approximated streamfunction is obtained, the momentum equations can be used to approximate the pressure.

The goal of this paper is to demonstrate an algorithm that uses the twolevel finite element method to approximate the streamfunction and pressure recovery 
The organization of the paper is as follows. Section 2 presents the model equations and the weak forms. Section 3 presents Pressure recovery algorithm and general analysis of convergence for this algorithm are presented in Section 4. Finally, in Section 4 we apply our method to one test problem.

\section{Governing Equations}

Consider the Navier-Stokes equations describing the flow of an incompressible fluid

$$
\begin{aligned}
& -\operatorname{Re}^{-1} \triangle \boldsymbol{u}+(\boldsymbol{u} \cdot \triangle) \boldsymbol{u}+\nabla p=\boldsymbol{f} \quad \text { in } \Omega, \\
& \nabla \cdot \boldsymbol{u}=0 \text { in } \Omega \text {, } \\
& \boldsymbol{u}=0 \quad \text { in } \partial \Omega,
\end{aligned}
$$

where $\boldsymbol{u}=\left(u_{1}, u_{2}\right)$ and $p$ denote, respectively, the unknown velocity and pressure field in a bounded, simply-connected polygonal domain $\Omega \subseteq R^{2} ; \boldsymbol{f}$ is a given body force, and Re is the Reynolds number.

The introduction of a stream function $\psi(x, y)$ defined by

$$
u_{1}=-\frac{\partial \psi}{\partial y}, \quad u_{2}=\frac{\partial \psi}{\partial x}
$$

means that the continuity condition (2) is satisfied identically. The pressure may then be eliminated from (1) to give

$$
\begin{aligned}
\operatorname{Re}^{-1} \triangle^{2} \psi_{y} \triangle \psi_{x}+\psi_{x} \triangle \psi_{y} & =\operatorname{curl} \boldsymbol{f} \text { in } \Omega, \\
\psi & =0 \text { on } \partial \Omega, \\
\frac{\partial \psi}{\partial \hat{n}} & =0 \text { on } \partial \Omega,
\end{aligned}
$$

where $\hat{n}$ represents the outward unit normal to $\Omega$. In order to write $(4)-(6)$ in a variational form, we define the Sobolev spaces

$$
\begin{aligned}
H^{1}(\Omega) & =\left\{v: v \in L^{2}(\Omega), D v \in L^{2}(\Omega)\right\}, \\
H_{0}^{1}(\Omega) & =\left\{v: v \in H^{1}(\Omega), v=0 \text { on } \partial \Omega\right\}, \\
H^{2}(\Omega) & =\left\{v: v \in L^{2}(\Omega), D v \in L^{2}(\Omega), D^{2} v \in L^{2}(\Omega)\right\}, \\
H_{0}^{2}(\Omega) & \left.=\left\{v: v \in H^{2}(\Omega): v=\frac{\partial v}{\partial n}=0 \text { on } \partial \Omega\right)\right\}, \\
{\left[H_{0}^{1}(\Omega)\right]^{2} } & =H_{0}^{1}(\Omega) \times H_{0}^{1}(\Omega), \\
H^{-m}(\Omega) & =\text { the dual of } H_{0}^{m}(\Omega)
\end{aligned}
$$

where $L^{2}(\Omega)$ is the space of square integrable functions on $\Omega$ and $D$ represents differentiations with respect to $x$ or $y$. For each $\phi \in H^{1}(\Omega)$, define curl $\phi=$ $\left(\begin{array}{r}\phi_{y} \\ -\phi_{x}\end{array}\right)$ 
The standard weak form of equations (1) - (3) is

Find $\boldsymbol{u} \in\left[H_{0}^{1}\right]^{2}, p \in L_{0}^{2}(\Omega)$, such that $\forall \boldsymbol{w} \in\left[H_{0}^{1}(\Omega)\right]^{2}, q \in L_{0}^{2}(\Omega)$

$$
\left.\begin{array}{rl}
\operatorname{Re}^{-1} \boldsymbol{a}(\boldsymbol{u}, \boldsymbol{w})+\tilde{b}(\boldsymbol{u} ; \boldsymbol{u}, \boldsymbol{w})+\tilde{c}(\boldsymbol{w}, p) & =\langle\boldsymbol{f}, \boldsymbol{w}\rangle \\
\tilde{c}(\boldsymbol{u}, q) & =0
\end{array}\right\}
$$

where

$$
\left.\begin{array}{rl}
\tilde{a}(\boldsymbol{u}, \boldsymbol{w}) & =\operatorname{Re}^{-1} \int_{\Omega} \nabla \boldsymbol{u}: \nabla \boldsymbol{w}, \\
\tilde{b}(\boldsymbol{u} ; \boldsymbol{v}, \boldsymbol{w}) & =\int_{\Omega}((\boldsymbol{u} \cdot \nabla) \boldsymbol{v}) \cdot \boldsymbol{w}, \\
\tilde{c}(\boldsymbol{w}, q) & =\int_{\Omega} q \operatorname{div} \boldsymbol{w},
\end{array}\right\}
$$

and $\langle\cdot, \cdot\rangle$ denotes the duality pairing in $L^{2}(\Omega)$.

The standard weak form of equations $(4)-(6)$ is

Find $\psi \in H_{0}^{2}(\Omega)$ such that for all $\phi \in H_{0}^{2}(\Omega)$,

$$
a(\psi \phi)+b(\psi ; \psi, \phi)=\ell(\phi),
$$

where

$$
\left.\begin{array}{rl}
a(\psi, \phi) & =\operatorname{Re}^{-1} \int_{\Omega} \triangle \psi \cdot \triangle \phi d \Omega, \\
b(\xi ; \psi, \phi) & =\int_{\Omega} \triangle \xi\left(\psi_{y} \phi_{x}-\psi_{x} \phi_{y}\right) d \Omega, \\
\ell(\phi) & =(\boldsymbol{f}, \operatorname{curl} \phi)=\int_{\Omega} \boldsymbol{f} \cdot \operatorname{curl} \phi d \Omega .
\end{array}\right\}
$$

\section{Pressure Recovery Algorithm}

We consider the approximate solution of (4) by a two-level, finite-element procedure. Let $X^{h}, X^{H} \subset H_{0}^{2}(\Omega)$ denote two conforming finite-element meshes with $H \gg h$. The method we consider computes an approximate solution $\psi^{h}$ in the finite-element space $X^{h}$ by solving one linear system for the degrees of freedom on $X^{h}$. This particular linear problem requires the construction of a finite-element space $X^{H}$ upon a very coarse mesh of width ' $H \gg h$ ', and then the solution of a much smaller system of nonlinear equations for an approximation in $X^{H}$.

Once the approximated $\psi^{h}$ is obtained, the momentum equations can be used to approximate the pressure. The basic idea is to solve an equation of the form

$$
\tilde{c}(\boldsymbol{v}, p)=g(\boldsymbol{v}),
$$

where

$$
\left.\begin{array}{rl}
g(\boldsymbol{v}) & =g(\psi ; \boldsymbol{f}, \operatorname{Re})(\boldsymbol{v}) \\
& =\langle\boldsymbol{f}, \boldsymbol{v}\rangle-\operatorname{Re}^{-1} \tilde{a}(\operatorname{curl} \psi, \boldsymbol{v})-\tilde{b}(\operatorname{curl} \psi ; \operatorname{curl} \psi, \boldsymbol{v}) .
\end{array}\right\}
$$


It is clear that $g$ depends on the solution $\psi$ of (13), the data $\boldsymbol{f}$ and Re. For any $g \in\left[H_{0}^{1}(\Omega)\right]^{\prime}$, a unique $p \in L_{0}^{2}(\Omega)$ exists such that (17) holds for each $\boldsymbol{v} \in\left[H_{0}^{1}(\Omega)\right]^{2}$ (see $[2]$ ). It follows directly from the continuity of $\tilde{a}$ and $\tilde{b}$ (see [10]) that

Lemma 1. For $\boldsymbol{f} \in \boldsymbol{H}^{-1}(\Omega), \psi \in H_{0}^{2}(\Omega), g$ as defined above is a bounded linear functional on $H_{0}^{1}(\Omega)$. Moreover, for all $\psi, \varphi \in H_{0}^{2}(\Omega), \boldsymbol{v} \in\left[H_{0}^{1}(\Omega)\right]^{2}$

$$
\left.\begin{array}{c}
|g(\psi ; \boldsymbol{f}, \operatorname{Re})(\boldsymbol{v})-g(\varphi ; \boldsymbol{f}, \operatorname{Re})(\boldsymbol{v})| \leq \\
\left(\operatorname{Re}^{-1}+\Gamma_{1}|\psi|_{2}+\Gamma_{1}|\varphi|_{2}\right)|\psi-\varphi|_{2}|\boldsymbol{v}|_{1} .
\end{array}\right\}
$$

Then we have

Theorem 1. Given $\psi \in H_{0}^{2}(\Omega), \boldsymbol{f} \in H^{-1}(\Omega)$, there exists a unique $p \in$ $L_{0}^{2}(\Omega)$ such that

$$
\tilde{c}(\boldsymbol{v}, p)=-g(\psi ; \boldsymbol{f}, \operatorname{Re})(\boldsymbol{v}) \text { for all } \boldsymbol{v} \in\left[H_{0}^{1}(\Omega)\right]^{2} .
$$

Note that $\tilde{c}$ is continuous and coercive on $\left[H_{0}^{1}(\Omega)\right]^{2} \times L_{0}^{2}(\Omega)([10]$, Theorem 3.7 , p. 35). The coercivity condition does not necessarily hold for arbitrary subspace $V^{h} \subset\left[H_{0}^{1}(\Omega)\right]^{2}$ and $S^{h} \subset L_{0}^{2}(\Omega)$. Also, in discretizing (17), not only will $P$ and $\boldsymbol{v}$ be discretized, but so will $g$. We mean that $g(\psi ; \boldsymbol{f}, \operatorname{Re})(\boldsymbol{v})$ has to be replaced by $g\left(\psi^{h} ; \boldsymbol{f}, \operatorname{Re}\right)\left(\boldsymbol{v}^{h}\right)$. Since the null space of $g\left(\psi^{h} ; f, \operatorname{Re}\right)$ does not necessarily coincide with the discretely div-free functions in $V^{h}$, the discretized analog of (17) can only hold in some subspace of $V^{h}$. Thus subspace is generally quite difficult to find. Because of this, Cayco and Nicolaides [5] introduced the following equivalent problem:

Find $\boldsymbol{w} \in\left[H_{0}^{1}(\Omega)\right]^{2}, p \in L_{0}^{2}(\Omega)$ such that for all $\boldsymbol{v} \in\left[H_{0}^{1}(\Omega)\right]^{2}, q \in L_{0}^{2}(\Omega)$

$$
\left.\begin{array}{rl}
\operatorname{Re}^{-1} \tilde{a}(\boldsymbol{w}, \boldsymbol{v})+\tilde{c}(\boldsymbol{v}, p) & =g(\psi ; \boldsymbol{f}, \operatorname{Re})(\boldsymbol{v}) \\
\tilde{c}(\boldsymbol{w}, q) & =0
\end{array}\right\}
$$

(20) is uniquely solvable (See [10]), and by Theorem $3.2, w=0$. We discretize (20) as follows:

Find $\boldsymbol{w}^{h} \in V^{h}, p^{h} \in S^{h}$ such that for all $\boldsymbol{v}^{h} \in V^{h}, q^{h} \in S^{h}$

$$
\left.\begin{array}{rl}
\operatorname{Re}^{-1} \tilde{a}\left(\boldsymbol{w} \cdot \boldsymbol{v}^{h}\right)+\tilde{c}\left(\boldsymbol{v}^{h}, p^{h}\right) & =g\left(\psi^{h} ; \boldsymbol{f}, \operatorname{Re}\right)\left(\boldsymbol{v}^{h}\right) \\
\tilde{c}\left(\boldsymbol{w}^{h}, q^{h}\right) & =0
\end{array}\right\}
$$

The solution procedure is then given as follows:

\section{Algorithm}

Step 1: Solve the nonlinear system on coarse mesh for $\psi^{H} \in X^{H}$

$$
a\left(\psi^{H}, \varphi^{H}\right)+b\left(\psi^{H}, \psi^{H}, \varphi^{H}\right)=\left(\boldsymbol{f}, \operatorname{curl} \varphi^{H}\right) \text { for all } \varphi^{H} \in X^{H} .
$$


Step 2: Solve the linear system on fine mesh for $\psi^{h} \in X^{h}$

$$
a\left(\psi^{h}, \varphi^{h}\right)+b\left(\psi^{H}, \psi^{h}, \varphi^{h}\right)=\left(\boldsymbol{f}, \operatorname{curl} \varphi^{h}\right) \text { for all } \varphi^{h} \in X^{h} .
$$

Step 3: Solve the linear system for $\boldsymbol{w}^{h} \in V^{h}, p^{h} \in S^{h}$ such that for all $\boldsymbol{v}^{h} \in$ $\overline{V^{h}, q^{h}} \in S^{h}$

$$
\left.\begin{array}{rl}
\operatorname{Re}^{-1} \tilde{a}\left(\boldsymbol{w}^{h}, \boldsymbol{v}^{h}\right)+\tilde{c}\left(\boldsymbol{v}^{h}, p^{h}\right) & =g\left(\psi^{h} ; \boldsymbol{f}, \operatorname{Re}\right)\left(\boldsymbol{v}^{h}\right) \\
\tilde{c}\left(\boldsymbol{w}^{h}, q^{h}\right) & =0
\end{array}\right\}
$$

Existence, uniqueness and error bound of Steps 1 and 2 are given in the following theorems. The proofs of these theorems are given in [8].

Theorem 2 (Step 1). (a) If the global uniqueness condition $\operatorname{Re}^{2} N|f|_{*}<1$ holds, $\psi$ and $\psi^{H}$ both exist uniquely, then the error $\left|\psi-\psi^{H}\right|_{2}$ satisfies

$$
\left|\psi-\psi^{H}\right|_{2} \leq C(\operatorname{Re}) \inf _{w^{H} \in X^{h}}\left|\psi-w^{H}\right|_{2},
$$

where

$$
\begin{aligned}
N & :=\sup _{\xi, \psi, \varphi \in H_{0}^{2}(\Omega)} \frac{|b(\xi, \psi, \varphi)|}{|\psi|_{2} \cdot|\varphi|_{2}} \\
|f|_{*} & :=\sup _{\varphi \in H_{0}^{2}(\Omega)} \frac{(f, \operatorname{curl} \varphi)}{|\varphi|_{2}} \\
C(\operatorname{Re}) & =\left(1+2 N|f|_{*} \cdot \operatorname{Re}^{2}\right)\left(1-N|f|_{*} \operatorname{Re}^{2}\right)^{-1} \leq C\left(\sqrt{N|f|_{*}}\right) .
\end{aligned}
$$

(b) If the uniqueness condition fails, and $\psi$ is a non-singular solution of (4), then there is an $H_{0}=H_{0}(\psi, f, \operatorname{Re})$ and $c=c(\psi, f, \operatorname{Re}, N)$ such that for $H \leq H_{0}$,

$$
\left|\psi-\psi^{H}\right|_{2} \leq c(\psi, f, \operatorname{Re}, N) \inf _{w^{H} \in X^{h}}\left|\psi-w^{H}\right|_{2},
$$

where $c(\psi, f, \operatorname{Re}, N)=\gamma^{-1}\left(\operatorname{Re}^{-1}+N \cdot \operatorname{Re}|f|_{*}\right)+1$.

Theorem 3 (Step 2). Let $X^{h, H} \subset H_{0}^{2}(\Omega)$ be two finite element spaces, $\psi$ a solution to (4) and $\psi^{H}$ a solution to (23). Then the solution to (24) exists uniquely and satisfies

$$
\left|\psi-\psi^{h}\right|_{2} \leq c_{1} \inf _{\varphi^{h} \in X^{h}}\left|\psi^{h}-\varphi^{h}\right|_{2}+c_{2} \sqrt{|\ln h|}\left|\psi-\psi^{H}\right|_{1},
$$

where $c_{1}=2+N|f|_{*} \operatorname{Re}^{2}$ and $c_{2}=2 N \cdot \operatorname{Re}^{2}|f|_{*} c$.

Our aim in this paper is to give an error analysis for Step 3. This analysis will be explained in the next section. 


\section{Main Result}

In this section, we establish an error estimate for Step 3 in the above algorithm. The following theorem is the main theorem in this paper which gives an error estimate.

Theorem 4. Let $\psi$ be the solution to $(4-6)$ and $\psi^{h}$ the solution to (24). Let the space $V^{h} \subset\left[H_{0}^{1}(\Omega)\right]^{2}$ and the space $S^{h} \subset L_{0}^{2}(\Omega)$ be chosen such that

$$
\tilde{C}\left(v^{h}, q^{h}\right) \geq \beta\left\|q^{h}\right\|_{0} \cdot\left|v^{h}\right| .
$$

Then (25) is uniquely solvable and

$$
\left\|p-p^{h}\right\|_{0} \leq K_{0}\left|\psi-\psi^{h}\right|_{2}+K_{1} \inf _{q^{h} \in S^{h}}\left\|p-q^{h}\right\|_{0},
$$

where $K_{0}, K_{1}$ are positive constants independent of $p, p^{h}, w^{h}, \psi$ and $\psi^{h}$.

Proof. . It follows from the primitive variable theory that (22) is uniquely solvable. Subtracting (21) from (22), we get

$$
\tilde{a}\left(w-w^{h}, v^{h}\right)=-\tilde{c}\left(v^{h}, p-p^{h}\right)+g(\psi ; f, \operatorname{Re})\left(v^{h}\right)-g\left(\psi^{h}, f, \operatorname{Re}\right)\left(v^{h}\right) .
$$

Also, we have

$$
\tilde{c}\left(w^{h}, p^{h}\right)=\tilde{c}\left(w^{h}, q^{h}\right)=0 \text { for all } q^{h} \in S^{h} .
$$

Setting $v^{h}=w^{h}$ in (30), we get

$$
\left|w^{h}\right|_{1} \leq \alpha_{1}\left|\psi-\psi^{h}\right|_{2}+\left\|p-q^{h}\right\|_{0},
$$

where $\alpha_{1}=\operatorname{Re}^{-1}+2\|f\| \operatorname{Re}^{2}$.

Now using (29) for any $q^{h} \in S^{h}$,

$$
\begin{aligned}
\beta\left\|q^{h}-p^{h}\right\| & \leq \sup _{v^{h} \in V^{h}} \frac{\left|\tilde{c}\left(v^{h}, q^{h}-p^{h}\right)\right|}{\left|v^{h}\right|_{1}} \\
& =\sup _{v^{h} \in V^{h}} \frac{\left|\tilde{c}\left(v^{h}, q^{h}-p\right)+\tilde{c}\left(v^{h}, p-p^{h}\right)\right|}{\left|v^{h}\right|_{1}} .
\end{aligned}
$$

Using (19), we get

$$
\begin{aligned}
& \beta\left\|q^{h}-p^{h}\right\|_{0} \leq \\
\leq & \sup _{v^{h} \in V^{h}} \frac{\left|\tilde{c}\left(v^{h}, q^{h}-p\right)-\tilde{a}\left(w-w^{h}, v^{h}\right)+g(\psi, f, \operatorname{Re})\left(v^{h}\right)-g\left(\psi^{h}, f, \operatorname{Re}\right)\left(v^{h}\right)\right|}{\left|v^{h}\right|_{1}} \\
\leq & \gamma_{1}\left\|q^{h}-p\right\|_{0}+\gamma_{2}\left|w-w^{h}\right|_{1}+\gamma_{3}\left|\psi-\psi^{h}\right|_{2},
\end{aligned}
$$


which implies

$$
\left\|q^{h}-p^{h}\right\|_{0} \leq \frac{\gamma_{1}}{\beta}\left\|q^{h}-p\right\|_{0}+\frac{\gamma_{2}}{\beta}\left|w^{h}\right|_{1}+\frac{\gamma_{3}}{\beta}\left|\psi-\psi^{h}\right|_{2} .
$$

Triangle inequalities and (31) give

$$
\begin{aligned}
\left\|p-p^{h}\right\|_{0} \leq & \left\|p-q^{h}\right\|_{0}+\left\|p^{q}-p^{h}\right\|_{0} \\
\leq & \left\|p-q^{h}\right\|_{0}+\frac{\gamma_{1}}{\beta}\left\|q^{h}-p\right\|_{0}+\frac{\gamma_{2}}{\beta}\left|w^{g}\right|_{1}+\frac{\gamma_{3}}{\beta}\left|\psi-\psi^{h}\right|_{2} \\
\leq & \left\|p-q^{h}\right\|_{0}+\frac{\gamma_{1}}{\beta}\left\|q^{h}-p\right\|_{0}+\frac{\gamma_{2}}{\beta} \alpha_{1}\left|\psi-\psi^{h}\right|_{2} \\
& \quad+\frac{\gamma_{2}}{\beta}\left\|q^{h}-p\right\|_{0}+\frac{\gamma_{3}}{\beta}\left|\psi-\psi^{h}\right|_{2} \\
\leq & K_{0}\left|\psi-\psi^{h}\right|_{2}+K_{1}\left\|q^{h}-p\right\|_{0},
\end{aligned}
$$

where $K_{0}=\frac{\gamma_{2}}{\beta} \alpha_{1}+\frac{\gamma_{3}}{\beta}$ and $K_{1}+1+\frac{\gamma_{1}}{\beta}+\frac{\gamma_{2}}{\beta}$.

Hence $\left\|p-p^{h}\right\|_{0} \leq K_{0}\left|\psi-\psi^{h}\right|_{2}+K_{1} \inf _{q^{h} \in S^{h}}\left\|q^{h}-p\right\|_{0}$.

\section{$5 \quad$ Numerical Examples}

In this section we describe some numerical results obtained by implementing the two-level algorithm for the well-known driven cavity problem. We chose this problem because there are numerous results in the literature with which to compare. Cavity flows have been a subject of study for some time. These flows have been widely used as test cases for validating incompressible fluid dynamics algorithm. Corner singularities for two-dimensional fluid flows are very important since most examples of physical interest have corners. For example, singularities of most elliptic problems develop when the boundary contour is not smooth. In this example, we consider the driven flow in a rectangular cavity when the top surface moves with a constant velocity along its length. The upper corners where the moving surface meets the stationary walls are singular points of the flow at which the horizontal velocity is multivalued. The lower corners are also weakly singular points.

For this problem, the region $\Omega$ is the unit square $\{0<x<1,0<$ $y<1\}$ with no-slip boundary conditions, i.e., $u=v=0$ in all boundaries except $y=1$, where $u=1$. This problem has been studied and addressed by many researchers including Ghia, Ghia, Shin [9], and J.E. Akin [1]. The numerical computation of this example was obtained using a Sun Ultra 2 with $2200 \mathrm{MHz}$ ultrasparc processor running Solaris 2.5.1. and for the finite element discretization we use the Bogner-Fox-Schmit elements. Bogner-FoxSchmit elements are bicubic polynomials within each rectangle. The degrees of freedom are chosen to be the function value, the first derivatives, and the 
mixed second derivative at the vertices. We set the function and the normal derivative values equal to zero at all vertices on the boundary. Bogner-FoxSchmit elements are used with $17 \times 17$ grid points on the coarse mesh and $33 \times 33$ grid points on the fine mesh. The streamlines for $\operatorname{Re}=1,10,50,100$ are plotted in Fig.1. All nonlinear problems were solved by Newton's method until the norm of the difference in successive iterates and the norm of residual were within a fixed tolerance. In each Newton's iteration, we need to solve a linear system. The resulting linear system is non-symmetric whose symmetric part is positive definite. Moreover, the resulting matrix is sparse matrix. We choose the Bi-Conjugate Gradient Stabilized method (BICGSTAB) which requires two matrix-vector products and four inner products in each iteration. BICGSTAB is given and discussed in [3]. When solving the linearized problem with a mesh spacing $h$ we need the solution $u^{H}$ generated on a mesh with spacing $H$. To do this we interpolate the solution $u^{H}$ onto the grid with spacing $h$.

\section{Acknowledgment}

The auther is greatful for the financial and facilities support provided by KFUPM.

\section{References}

1. J. Akin. finite element for analysis and design. Academic Press, San Diego, 1994.

2. I. Babuska and A. K. Aziz. The Mathematical Foundations of the Finite Element Method with Applications to Partial Differential Equations. Academic Press, New York, 1972.

3. R. Barrett, M. Berry T. Chan J. Demmel J. Donator J. Doncarra V. Eijkhout R. Pozo C. Romine and H. Van dev Vorst, Templates: for the solution of linear systems: building blocks for iterative methods. e-mail : templates@cs.utk.edu.

4. M. Cayco. Finite Element Methods for the Stream Function Formulation of the Navier-Stokes Equations. PhD thesis, CMU, Pittsburgh, PA., 1985.

5. M. Cayco and R.A. Nicolaides. Analysis of nonconforming stream function and pressure finite element spaces of the Navier-Stokes equations. Comp. and Math. Appl., (8): 745-760, 1989.

6. M. Cayco and R.A. Nicolaides. Finite element technique for optimal pressure recovery from stream function formulation of viscous flows. Math. Comp., (56): 371-377, 1986.

7. Ph. G. Ciarlet, the finite element method for elliptic problems, North Holland, Amsterdam, 1978

8. F. Fairag. Two-level finite element method for the stream function formulation of the Navier-Stokes equations. Computers Math. Applic., 36(2): 117-127, 1998.

9. K.N. Ghia U. Ghia and C.T. Shin. High Re solutions for incompressible flow using the Navier-Stokes equations and a multigrid method. J. Comput. Phys., 48: 387-411, 1982. 
10. V. Girault and P. A. Raviart. Finite Element Approximation of the NavierStokes Equations, volume 749. Springer, Berlin, 1979.

11. W. Layton. A two-level discretization method for the Navier-Stokes equations. Computers Math. Applic., 26:33-38, 1993.

12. W. Layton and W. Lenferink. Two-level Picard-defect corrections for the Navier-Stokes equations at high Reynolds number. Applied Math. and Computing, 1995.

13. W. Layton and W. Lenferink, A multilevel mesh independence principle for the Navier-Stokes equations, SIAM J. N. A. (1996)

14. J. Xu. A novel two-grid method for semilinear elliptic equations. SIAM J. Sci. Comput. 15(1): 231-237, 1994.

15. J. Xu, Some Two-Grid Finite Element Methods, chapter 157, pages 79-87. Number 157 in In Domain Decomposition Methods in Science and Engineering. Amer. Math. Soc., Providence, RI, 1994.

16. J. Xu. Some two-grid finite element methods. Technical report, P. S.U., 1992.

17. X. Ye. Two-level discretizations of the stream function form of the NavierStokes equations. University of Pittsburgh. 


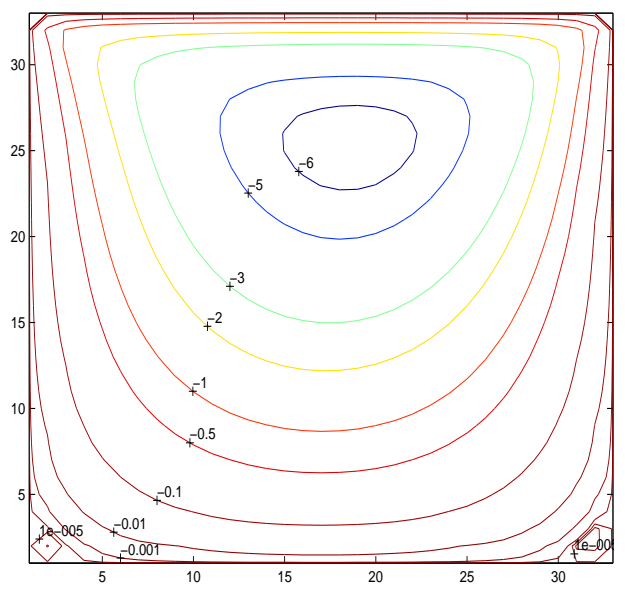

(a) $\operatorname{Re}=1$

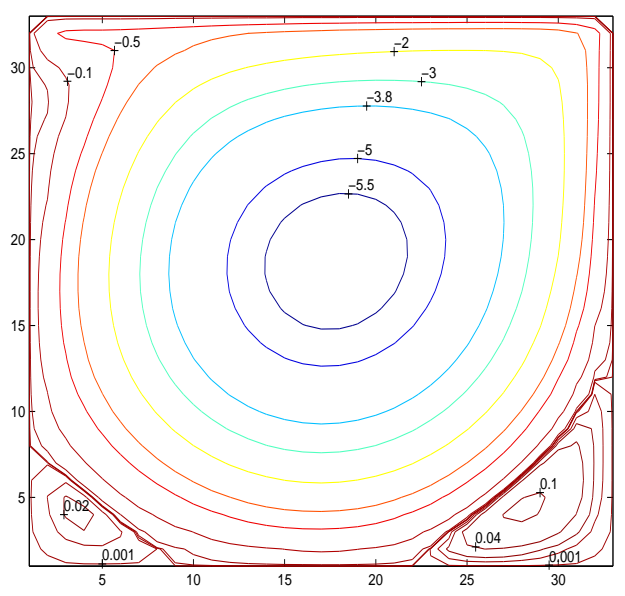

(c) $\operatorname{Re}=50$

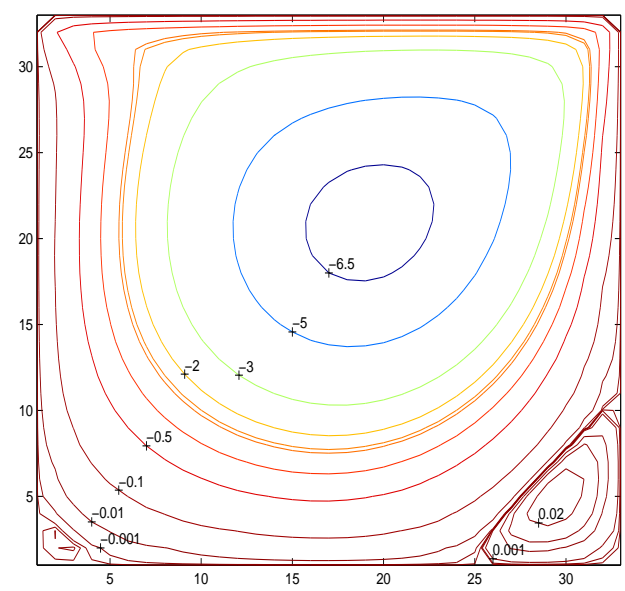

(b) $\operatorname{Re}=10$

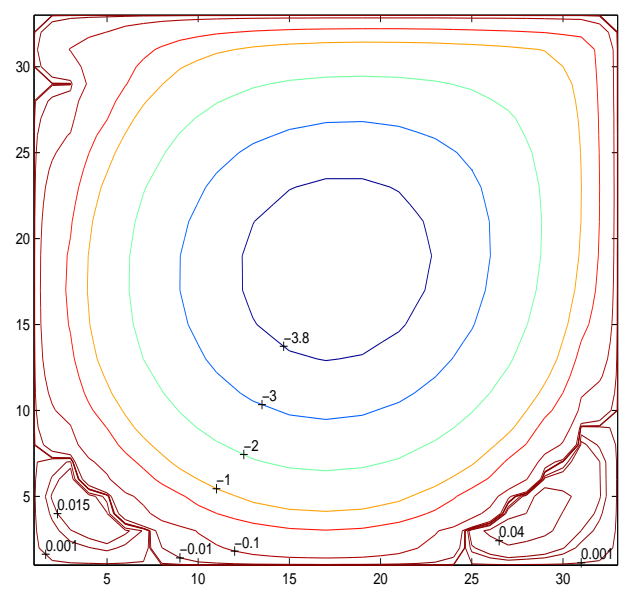

(d) $\operatorname{Re}=100$

Fig. 1. Cavity Problem : Streamlines for $H=\frac{1}{16}, h=\frac{1}{32}$ with different values of Re numbers using two level method 
